misch von Tryptophan und Cystin, das zuerst infolge Einwirkung von UV-Licht vergilbt war, bei nachheriger Bestrahlung mit Licht der Wellenlänge 410-430 mu kein Bleicheffekt.

$$
\text { A. Sippel (Freiburg i. Br.) }
$$

Nanjundayya, C. (The Bombay Textile Res. Assoc., Ghatkopar, Bombay, Indien). Die Festigkeit von Baumwollgarn mit besonderer Berïcksichtigung der Struktur im Bereich des Bruches. (Textile Res. J. 36, 954-966, 1966.)

Baumwollgarne reißen vorzugsweise dort, wo sie am dünnsten bzw. wo die Drehung am höchsten ist. Zählt man die an dieser Stelle tatsächlich gerissenen Fasern, so kommt man auf höhere Werte der Bruchfestigkeit, als in der Literatur angegeben ist. Die gerissenen Fasern haben einen Titer, der etwas unter dem Mittelwert liegt.

$$
\text { A. Sippel (Freiburg i. Br.) }
$$

Patil, N. B. u. T. Radhakrishnan (The Ahmedabad Textile Industr. Res. Assoc., Ahmedabad, Indien). Studien über die Entkristallisierung von Baumwolle. Kristalline und amorphe Cellulosestandards. (Textile Res. J. 36, 1043-1050, 1966.)

Es wird gefunden, daß in der Kugelmühle gemahlene Cellulose als Standard für amorphe Cellulose befriedigt. Wenn man vermittels Hydrolyse ein möglichst kristallines Material herzustellen sucht, so gelingt dies bei Ramie besser als bei Baumwolle. A. Sippel (Freiburg i. Br.)

\section{c) Textilveredlung}

Feldtman, H. D., J. R. McPhee u. A. J. Pratt (Div. of Textile Industry, C.S.I.R.O. Wool Res. Labors., Geelong, Victoria, Australien). Behandlung von Wolle mit Polyolefinen. (Textile Res. J. 37, 46-53, 1967.)

Wolle kann durch Aufbringen von Polyäthylen oder Polyvinylacetat krumpffest gemacht werden, wenn man durch Anwendung von UV-Bestrahlung oder von Benzoylperoxid für Vernetzung sorgt. Bei Benutzung von Polypropylen anstelle von Polyäthylen gelingt dies nicht.

$$
\text { A. Sippel (Freiburg i. Br.) }
$$

Griffith, J. C. u. P. Mason (Div. of Textile Physies, C.S.I.R.O. Wool Res. Labors., Ryde, Sydney, Australien). Die Auswirkung von Formaldehyd auf Wolle vor, während und nach einer Kontraktion in Lithiumbromid-Lösung. (Textile Res. J. 36, 1021-1022, 1966.)

Wird eine in Lithiumbromidlösung voll kontrahierte Faser mit Formaldehyd behandelt, so erfährt sie eine um $33 \%$ höhere Zunahme des Elastizitätsmoduls als beim Blindversuch, wodurch
Vernetzung nachgewiesen ist. Erfolgt die Formaldehydbehandlung vor der Lithiumbromidbehandlung, so wird hierdurch das Ausmaß der Kontraktion vermindert. Auch bei gleichzeitiger Vornahme der Lithiumbromid- und Formaldehydbehandlung wird der Verlauf der Kontraktion sehr gestört, vor allem zeigt das Kraft-DehnungDiagramm ein völlig abweichendes Verhalten.

$$
\text { A. Sippel (Freiburg i. Br.) }
$$

Iwanow, N. u. R. Schneider (Centre de Recherches Textiles, Mulhouse, Frankreich). Studium der Diffusions- und Adsorptionsphänomene von Direktfarbstoffen bei Viskosefasern mittels der Methode der untersehiedlichen Anfärbung II. Eine statistische Studie über Faserpopulationen. (Textile Res. J. $37,11-40,1967$.)

Eine schon früher von den Autoren benutzte Färbetechnik (Textile Res. J. 26, 407, 1956) wird verfeinert und auf große Faserkollektive angewendet. Erst die statistische Auswertung gestattet, reproduzierbar die Einflüsse der Herstellungsweise sowie der Verarbeitung nachzuweisen. Das Verfahren ist wichtig zur Untersuchung streifiger Färbungen.

$$
\text { A. Sippel (Freiburg i. Br.) }
$$

McPhee, J. R. (Div. of Textile Industr., C.S.I.R.O., Wool Res. Labors. Geelong, Vict., Australien). Mottenfestmachen von Wolle mittels bromhaltiger organischer Verbindungen. (Textile Res. J. 36, 941-946, 1966.)

Nach Prüfung zahlreicher bromhaltiger organischer Verbindungen wird ein Gemisch bromierter Salicylanilide (,Diaphene") und 5-Brommethyl$1,2,3,4,7,7$ - hexachloro - 1,4 - methano-cyclohex - 2 -en (Bromodan) als Mottenschutzmittel mit geringer Toxizität gegenüber Säugetieren empfohlen. A. Sippel (Freiburg i. Br.)

\section{Berichtigung}

zur Arbeit

\section{Elektronenmikroskopische Untersuchungen an Oberflächen von Membranfiltern}

\section{Von H. J. Preußer-Darmstadt}

Kolloid-Z. und Z. Polymere 218, 129 (1967).

Durch ein Versehen wurden die Abbildungen $3 a$ und $3 \mathrm{~b}$ vertauscht.

Für die Schriftleitung verantwortlich: Für die Originalarbeiten Prof. Dr. F.H. Müller, 3550 Marbach b. Marburg/L. und für Referate und Berichte Dr. E. Uthlein, 6000 Frankfurt/M.

Anzeigenverwaltung: Dr. Karl Niedermeyer, 6000 Frankfurt/M. -West, Georg-Speyer-Straße 76

Dr. Dietrich Steinkopff Verlag, 6100 Darmstadt, Saalbaustraße 12 Satz und Druck: Universitätsdruckerei Mainz GmbH 